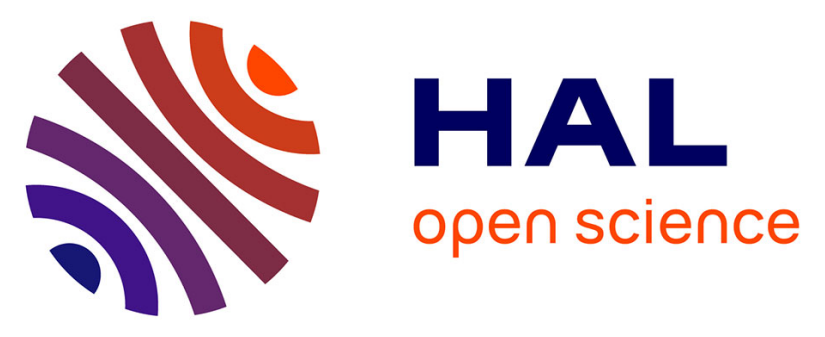

\title{
Efficient coupler between silicon photonic and metal-insulator-silicon-metal plasmonic waveguides
}

Alexandros Emboras, R. Briggs, A. Najar, S. Nambiar, Cécile Delacour, P. Grosse, E. Augendre, J. Fedeli, B. de Salvo, Ha Atwater, et al.

\section{To cite this version:}

Alexandros Emboras, R. Briggs, A. Najar, S. Nambiar, Cécile Delacour, et al.. Efficient coupler between silicon photonic and metal-insulator-silicon-metal plasmonic waveguides. Applied Physics Letters, 2012, 101 (25), pp.251117. 10.1063/1.4772941 . hal-00992898

\section{HAL Id: hal-00992898 \\ https://hal.science/hal-00992898}

Submitted on 19 May 2014

HAL is a multi-disciplinary open access archive for the deposit and dissemination of scientific research documents, whether they are published or not. The documents may come from teaching and research institutions in France or abroad, or from public or private research centers.
L'archive ouverte pluridisciplinaire HAL, est destinée au dépôt et à la diffusion de documents scientifiques de niveau recherche, publiés ou non, émanant des établissements d'enseignement et de recherche français ou étrangers, des laboratoires publics ou privés. 


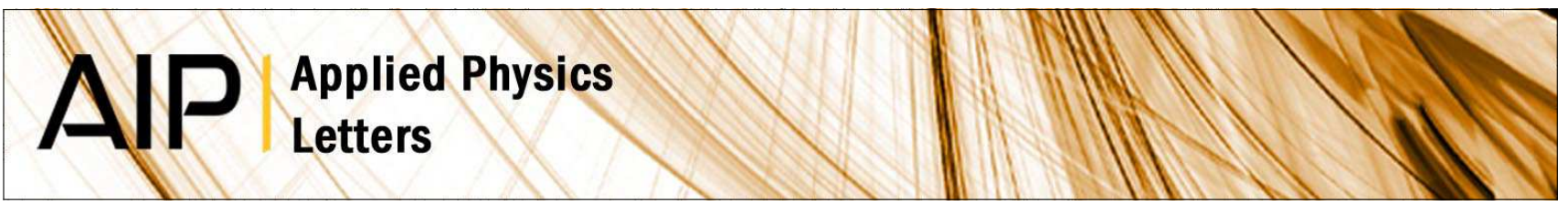

\title{
Efficient coupler between silicon photonic and metal-insulator-silicon-metal plasmonic waveguides
}

\author{
A. Emboras, R. M. Briggs, A. Najar, S. Nambiar, C. Delacour et al.
}

Citation: Appl. Phys. Lett. 101, 251117 (2012); doi: 10.1063/1.4772941

View online: http://dx.doi.org/10.1063/1.4772941

View Table of Contents: http://apl.aip.org/resource/1/APPLAB/v101/i25

Published by the American Institute of Physics.

\section{Related Articles}

Nonlinear response of an ultracompact waveguide Fabry-Pérot resonator

Appl. Phys. Lett. 102, 011133 (2013)

Exciton polaritons in semiconductor waveguides

Appl. Phys. Lett. 102, 012109 (2013)

Photonic microdisk resonators in aluminum nitride

J. Appl. Phys. 113, 016101 (2013)

Electrically controlled absorption in a slab waveguide formed by the implantation of protons in a potassium lithium tantalate niobate substrate

Appl. Phys. Lett. 101, 261101 (2012)

$\mu$-Raman spectroscopy characterization of LiNbO3 femtosecond laser written waveguides

J. Appl. Phys. 112, 123108 (2012)

\section{Additional information on Appl. Phys. Lett.}

Journal Homepage: http://apl.aip.org/

Journal Information: http://apl.aip.org/about/about_the_journal

Top downloads: http://apl.aip.org/features/most_downloaded

Information for Authors: http://apl.aip.org/authors

\section{ADVERTISEMENT}

\section{AIP Applied Physics Letters}

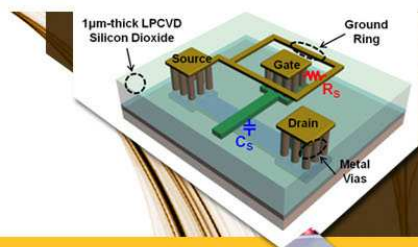

SURFACES AND INTERFACES

Focusing on physical, chemical, biological structural, optical, magnetic and electrical properties of surfaces and interfaces, and more...

\section{EXPLORE WHAT'S NEW IN APL}

SUBMIT YOUR PAPER NOW!
ENERCY CONVERSION AND STORACE 


\title{
Efficient coupler between silicon photonic and metal-insulator-silicon-metal plasmonic waveguides
}

\author{
A. Emboras, ${ }^{1}$ R. M. Briggs,${ }^{2,3}$ A. Najar, ${ }^{1}$ S. Nambiar, ${ }^{1}$ C. Delacour, ${ }^{1}$ Ph. Grosse,${ }^{1}$ \\ E. Augendre, ${ }^{1}$ J. M. Fedeli, ${ }^{1}$ B. de Salvo, ${ }^{1}$ H. A. Atwater, ${ }^{2}$ and R. Espiau de Lamaestre ${ }^{1, a)}$ \\ ${ }^{1}$ CEA, LETI, MINATEC Campus, 17 rue des martyrs, Grenoble, France \\ ${ }^{2}$ Thomas J. Watson Laboratories of Applied Physics, California Institute of Technology, 1200 East California \\ Blvd., MC 128-95 Pasadena, California 91125, USA \\ ${ }^{3}$ Jet Propulsion Laboratory, California Institute of Technology, Pasadena, California 91109, USA
}

(Received 7 September 2012; accepted 6 December 2012; published online 21 December 2012)

\begin{abstract}
We report the experimental realization of a compact, efficient coupler between silicon waveguides and vertical metal-insulator-silicon-metal (MISM) plasmonic waveguides. Devices were fabricated using complementary metal-oxide-silicon technology processes, with copper layers that support low-loss plasmonic modes in the MISM structures at a wavelength of $1550 \mathrm{~nm}$. By implementing a short $(0.5 \mu \mathrm{m})$ optimized metal-insulator-silicon-insulator structure inserted between the photonic and plasmonic waveguide sections, we demonstrate experimental coupling loss of $2.5 \mathrm{~dB}$, despite the high optical confinement of the MISM mode and mismatch with the silicon waveguide mode. C 2012 American Institute of Physics. [http://dx.doi.org/10.1063/1.4772941]
\end{abstract}

As the dimensions of complementary metal-oxide-semiconductor (CMOS) electronic devices are scaled down, circuit time delays and power dissipation of on-chip interconnections are substantially increased. Silicon optical interconnect technologies could offer higher bandwidth and reduced power consumption. ${ }^{1}$ However, due to the diffraction limit of light at telecommunication wavelengths, optical components are generally large relative to CMOS electronic devices. Furthermore, weak electrooptical effects in silicon ${ }^{2}$ lead to a large footprint for silicon-based optical modulators, ${ }^{3}$ which could limit the applications of photonic integrated circuits. By overcoming the diffraction limit and enhancing the interaction of light with charge carriers in silicon, hybrid plasmonic devices can push optics further toward the length scale of electronics. ${ }^{4}$

Metal-insulator-silicon-metal (MISM) hybrid plasmonic waveguides are of particular interest for active CMOS plasmonics because they support highly confined modes $^{5}$ and can potentially be integrated with standard processes used in silicon-based electronics foundries. ${ }^{6}$ However, such plasmonic structures must balance high field confinement with optical losses. Minimizing metal-related optical losses for integrated plasmon-assisted devices remains a key issue that can be addressed through both material selection ${ }^{7}$ and careful design of waveguide geometries. Considering the latter, the mode size mismatch and difference in phase index between standard silicon-on-insulator (SOI) photonic waveguides and metal-insulator-metal waveguides lead to high coupling losses. To overcome this issue, several coupling configurations are already proposed in the literature, including optimized butt couplers, ${ }^{8,9}$ taper couplers, ${ }^{10}$ and directional couplers. ${ }^{11}$

In this article, we demonstrate a modified butt coupling scheme employing copper-based metal-insulator-silicon-insulator (MISI) waveguide sections between SOI and MISM plasmonic waveguides. We use CMOS technology to fabricate the coupled waveguides, and improved coupling

${ }^{\text {a)} E l e c t r o n i c ~ m a i l: ~ r o c h . e s p i a u-d e-l a m a e s t r e @ c e a . f r . ~}$ efficiency is experimentally demonstrated. The MISM structure used here is designed for electro-optical modulation; ${ }^{5}$ however, this paper focuses specifically on minimizing optical coupling loss, which is essential for demonstrating the viability of plasmonic components in photonic integrated circuits.

The asymmetric plasmonic-photonic hybrid structure investigated in this paper is schematically depicted in Fig. 1. It consists of a vertical MISM structure composed of two copper slabs and a silicon waveguide core with a thin gate

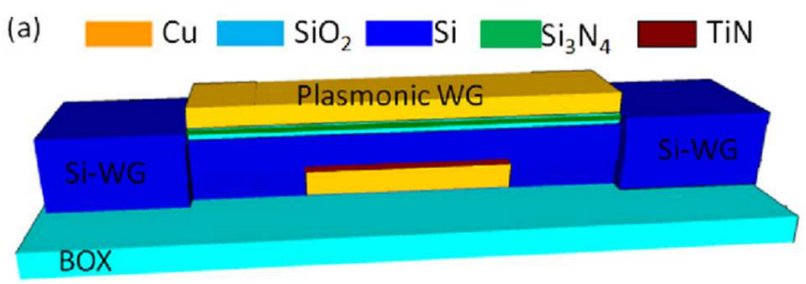

(b)

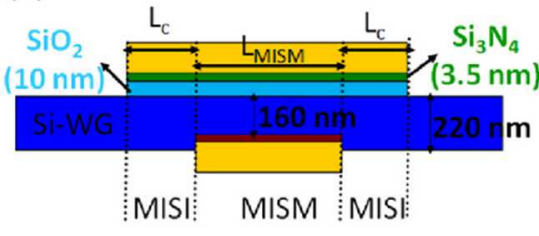

(d)

(c)
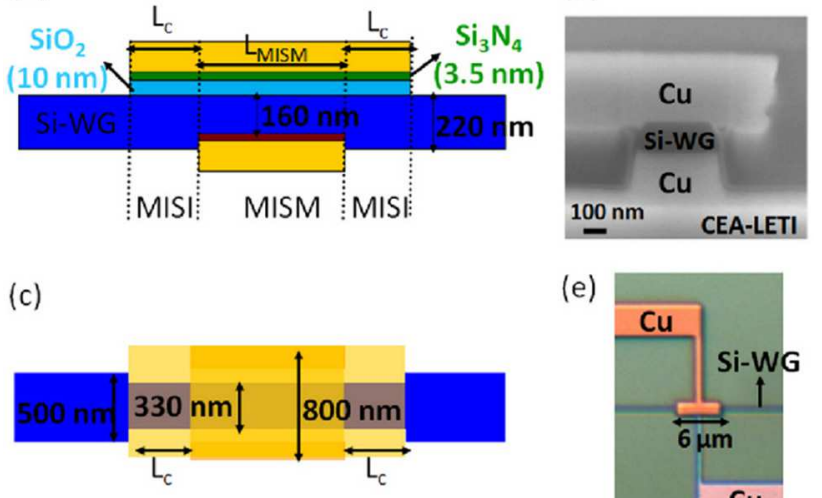

(e)

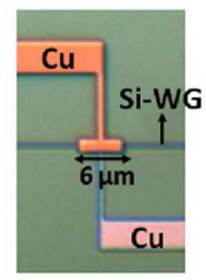

FIG. 1. (a) Three-dimensional view of the investigated hybrid plasmonic waveguide. (b) Side and (c) top views showing the coupling structure (MISI) connecting the SOI and plasmonic (MISM) waveguide sections. (d) SEM image of the cross section of the MISM plasmonic waveguide. (e) Optical microscope image (top view) of the fabricated plasmonic structure integrated with silicon waveguides. 
dielectric layer at one of the copper interfaces. The MISM structure is butt-coupled to a single-mode $\mathrm{Si}$ waveguide with a $220 \mathrm{~nm} \times 500 \mathrm{~nm}$ cross section through a compact coupling scheme composed of a vertical copper-insulator-silicon-insulator waveguide. This MISI section can be simply seen as the extension of the MIS structure of the MISM waveguide.

To investigate the optical losses of the devices, we first present results from three-dimensional electromagnetic finite-difference time-domain (FDTD) simulations, using the optical constants for silicon, $\mathrm{SiO}_{2}$ and $\mathrm{Si}_{3} \mathrm{~N}_{4}$ given by Palik ${ }^{12}$ and a copper refractive index of $0.20+11.10 i$ at $1550 \mathrm{~nm}$, based on spectroscopic ellipsometry measurements. We also model a $5 \mathrm{~nm}$ TiN layer at the interface between the silicon core and the back copper contact of the MISM waveguide section, which was implemented as a standard CMOS diffusion barrier, with a measured index of $3.29+5.90 i$. First, we investigate the silicon waveguide directly coupled to a MISM plasmonic waveguide of length $L_{\text {MISM }}$. Optical transmission for different values of $L_{\mathrm{MISM}}$ is shown in Fig. 2(b), where we observe an expected average increase in attenuation as $L_{\mathrm{MISM}}$ is increased, with an additional oscillatory behavior that will be discussed below. A linear fit (on a logarithmic loss scale) agrees with the calculated propagation loss of the fundamental plasmonic mode supported in the MISM waveguide, which was found to be $2.7 \mathrm{~dB} / \mu \mathrm{m}$ based on mode analysis of the two-dimensional waveguide cross section. By extrapolating to $L_{M I S M}=0$, we find a $3.1 \mathrm{~dB}$ per facet coupling loss. This relatively high value mainly originates from the mismatch between the mode size and effective index of the photonic and plasmonic modes supported in the silicon and MISM waveguides, which leads to back reflections into the SOI waveguide and scattering into radiative modes (14\% and 55\%, respectively, according to the three-dimensional simulations).

The FDTD calculation is consistent with the results of coupling theory ${ }^{1,13}$ using simple two-dimensional mode calculations. The Fresnel transmission coefficient, $T_{\text {SOI-MISM }}^{\text {Fresnel }}$, between the MISM fundamental plasmonic mode $\left(n_{\text {eff }}=3.43\right)$ and the TM-like silicon waveguide mode $\left(n_{\mathrm{eff}}=1.92\right)$ is 0.92 , but the electric field overlap integral is only $\Gamma_{\text {SOI-MISM }}=0.50$, since the field of the plasmonic mode is strongly confined to the thin insulator layer. We therefore estimate a power coupling loss into the MISM waveguide of $10 \log \left(T_{\text {SOI-MISM }}^{\text {Fresnel }} \Gamma_{\text {SOI-MISM }}\right)=3.4 \mathrm{~dB}$, in reasonable agreement with the numerical FDTD calculations.

This coupling efficiency can be enhanced by inserting an intermediate MISI section between the SOI and MISM waveguides. Figure 2(c) shows the calculated power attenuation at a wavelength of $\lambda=1550 \mathrm{~nm}$ through a $2.5 \mu \mathrm{m}$ long MISM waveguide with identical input and output MISI couplers of length $L_{\mathrm{c}}$. The total average attenuation, including the losses of both input and output MISI couplers, increases linearly with increasing $L_{\mathrm{c}}$, with a half slope of $0.185 \mathrm{~dB} /$ $\mu \mathrm{m}$, which is consistent with the mode loss $(0.165 \mathrm{~dB} / \mu \mathrm{m})$ of the fundamental plasmonic mode supported by the MISI waveguide. We also observe a strong oscillation behavior, and the attenuation is minimized near $L_{\mathrm{c}}=0.5 \mu \mathrm{m}$, with overall loss significant below the level calculated for the direct coupling configuration $\left(L_{\mathrm{c}}=0\right)$. Even though the optimized MISI couplers contribute an additional $2 L_{\mathrm{c}}=1 \mu \mathrm{m}$ to the overall length of the plasmonic waveguide section, introducing $0.165 \mathrm{~dB}$ of additional propagation loss, we note that the end-to-end attenuation is reduced by $4.8 \mathrm{~dB}$ compared to the direct coupling geometry.

Figure 2(b) also shows the power attenuation as a function of $L_{\mathrm{MISM}}$ for $L_{\mathrm{c}}=0.5 \mu \mathrm{m}$. Using this optimized coupling configuration, both the average attenuation and the oscillations in the attenuation as a function of $L_{\text {MISM }}$ are minimized. We note that by extending the copper back contact instead of the gate metal, in order to form a metal-silicon-insulator (MSI) coupling section, we observe no significant reduction in the overall coupling loss for small MSI lengths $\left(L_{\mathrm{MSI}}<1 \mu \mathrm{m}\right)$, and a dramatic increase in coupling loss increase for $L_{\mathrm{MSI}}>1 \mu \mathrm{m}$. This behavior is attributed to the high optical loss of the plasmonic mode supported at the copper-TiN-silicon interface. (a)

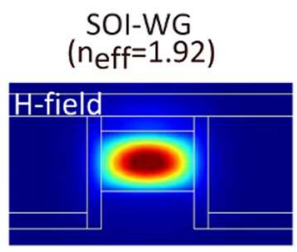

(b)

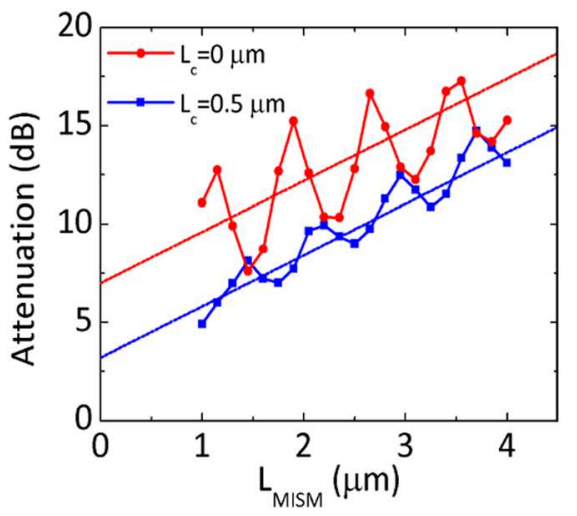

MISI-WG

$\left(n_{\text {eff }}=2.51\right)$

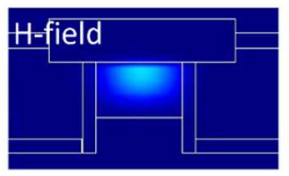

(c)

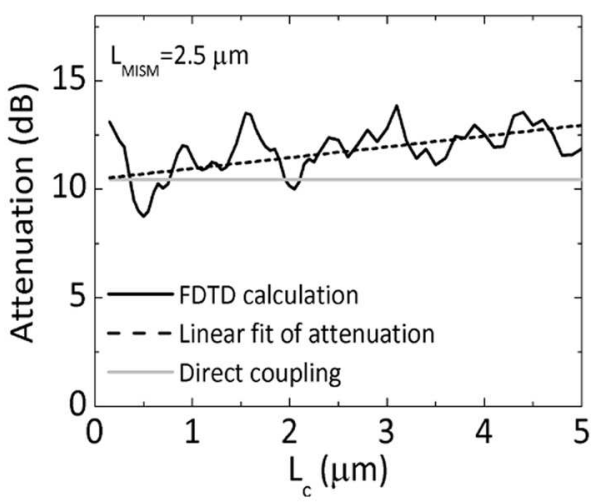

MISM-WG

$\left[n_{\text {eff }}=3.43\right)$

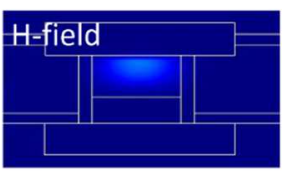

FIG. 2. (a) The $\mathrm{H}$ field intensity of the fundamental plasmonic mode supported in $\mathrm{Si}$ WG (left), in MISI plasmonic WG (middle) and in MISM plasmonic WG (right) taken from 3D mode analysis using FDTD (b) FDTD calculations of the power attenuation through a device having a constant $L_{\mathrm{c}}$. The dashed lines are the best fit with a slope fixed by the calculated propagation losses of the plasmonic mode supported by the MISM stack. (c) Power attenuation through a device having a constant $L_{\mathrm{MISM}}=2.5 \mu \mathrm{m}$ as function of the coupler length, $L_{\mathrm{c}}$ (black solid line). The dashed line is the best fit with a slope fixed by the propagation losses of the plasmonic mode of the MISI stack, and the gray line shows the loss for the direct coupling configuration $\left(L_{\mathrm{c}}=0\right)$. 
Plasmonic MISM waveguides were fabricated on a $220 \mathrm{~nm}$ SOI platform using an 8-in. diameter wafer CMOS fabrication facility. Light was coupled in and out of the silicon waveguides using diffraction grating couplers, which were fabricated by partially etching the SOI device layer. ${ }^{14}$ The grating dimensions $(950 \mathrm{~nm}$ period, $70 \mathrm{~nm}$ depth, and $50 \%$ duty cycle) were chosen so that the fundamental TM mode of the silicon waveguide could be accessed at a $1550 \mathrm{~nm}$ operating wavelength using a cleaved fiber positioned above the plane of the waveguide devices. The silicon waveguides were fabricated by photolithography and dry etching through the SOI device layer, and they were subsequently encapsulated with a conformal $60 \mathrm{~nm}$ thick $\mathrm{Si}_{3} \mathrm{~N}_{4}$ layer deposited by low-pressure chemical vapor deposition (LPCVD) and a thin silicon oxide layer.

For the MISM sections, the gate contact was patterned by a Damascene technique. After etching an opening through the encapsulation layer down to the silicon waveguide core, the insulator gate stack was formed by growing a $10 \mathrm{~nm}$ thermal oxide and depositing a $3 \mathrm{~nm} \mathrm{Si} \mathrm{Si}_{4}$ by LPCVD, which serves as a low optical loss copper diffusion barrier. ${ }^{6}$ A thick $(1.5 \mu \mathrm{m})$ copper layer was then deposited by sputtering a seed layer followed by electro-chemical deposition. Following metal deposition, the wafers were annealed, which improves the crystalline quality of the copper and therefore leads to reduced surface-plasmon propagation loss. ${ }^{15}$ After encapsulation of the gate contact with silicon nitride and silicon oxide using plasma-enhanced chemical vapor deposition, the wafers were planarized using chemical mechanical polishing. The waveguides were then transferred onto an oxidized bulk silicon wafer via direct bonding at atmospheric pressure and room temperature, with $\mu \mathrm{m}$-range control of wafer alignment. The bonded wafer pair was subsequently annealed at $200^{\circ} \mathrm{C}$ for $2 \mathrm{~h}$ to convert hydrogen bonds into covalent bonds and increase the bonding energy, and the SOI silicon handle was removed by mechanical lapping and chemical etching. Finally, the back metal contact was fabricated on the flipped structure, using a copper electro-chemical deposition on a $5 \mathrm{~nm}$ TiN diffusion barrier. An optical microscope image (top view) of the final device is shown in Fig. 1(e).

Automated measurement of optical transmission of the MISM devices was implemented at a wafer level scale using a $1550 \mathrm{~nm}$ laser source. To avoid back reflections, the light was coupled into the silicon waveguide grating couplers using a single-mode fiber positioned at an angle of $10^{\circ}$ with respect to the wafer surface normal. A multimode fiber was positioned at the output gratings and the transmission through the whole device was measured using an optical multimeter (Ando AQ2140). A modified Karl Suss automatic wafer probe station was used to switch from device to device on the wafer. The data were normalized to the optical transmission of reference $220 \mathrm{~nm} \times 500 \mathrm{~nm}$ silicon waveguides that were patterned alongside the waveguides with plasmonic MISM sections. Figure 3 shows the attenuation of the transmitted power of the plasmonic waveguides as a function of the MISM length, $L_{\mathrm{MISM}}$, for three different coupling lengths: $L_{\mathrm{c}}=0.3 \mu \mathrm{m}, L_{\mathrm{c}}=0.5 \mu \mathrm{m}$, and $L_{\mathrm{c}}=0.7 \mu \mathrm{m}$. The direct coupling case $\left(L_{\mathrm{c}}=0\right)$ is also presented.

We were able to test a significant number of devices owing to the large-scale CMOS fabrication techniques
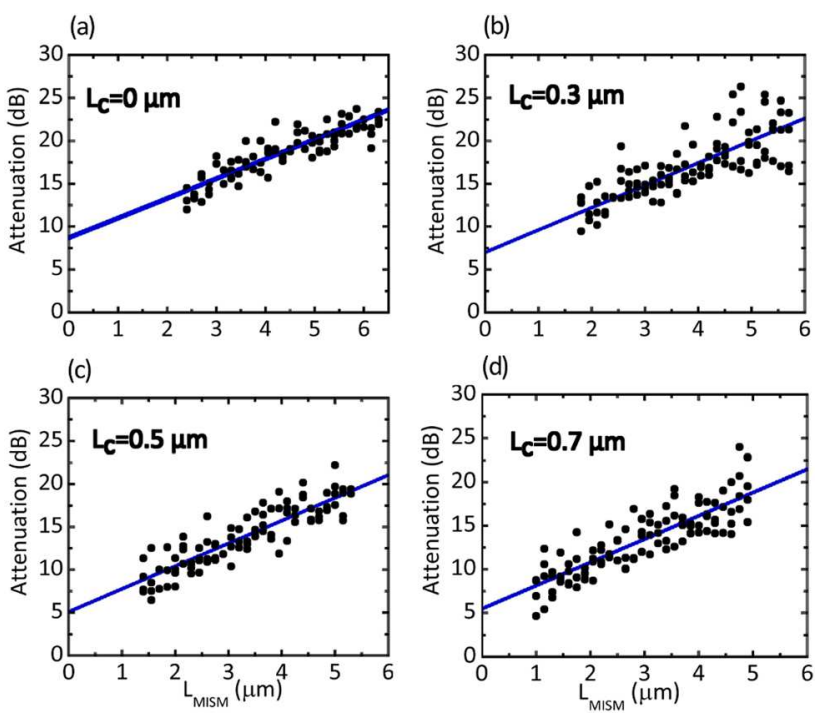

FIG. 3. Experimental transmission measurements as a function of the MISM length, $L_{\mathrm{MISM}}$, for different coupling lengths: (a) $\mathrm{L}_{c}=0 \mu \mathrm{m}$, (b) $\mathrm{L}_{c}=0.3 \mu \mathrm{m}$, (c) $L_{c}=0.5 \mu \mathrm{m}$, (d) $L_{c}=0.7 \mu \mathrm{m}$. In each figure, we show the experimental data (black dots) with a linear fit (blue line).

employed for this work, and the transmitted power of waveguides with nominally identical MISM sections were measured at three different positions on a completed wafer. The transmission data from all the devices were fit simultaneously, resulting in accurate estimates of the propagation and coupling losses. The fitted value for propagation loss is comparable for all values of $L_{\mathrm{c}}$, with an average value of $2.6 \pm 0.16 \mathrm{~dB} / \mu \mathrm{m}$, in agreement with the three-dimensional electromagnetic simulations. This provides convincing experimental verification that the transmission through the device is dominated by the fundamental plasmonic mode of the MISM stack. As with the numerical calculations, the coupling efficiency of the MISI section is determined experimentally by extrapolating the attenuation curve to $L_{\text {MISM }}=0$. These experimental coupling losses are reported in Fig. 4 together with the calculated values for different values of $L_{\mathrm{c}}$. We find that a coupling length of $0.5 \mu \mathrm{m}$ results in an optimal experimental coupling loss of $2.5 \pm 0.3 \mathrm{~dB}$, which is approximately half the loss measured for the direct coupling configuration. As a function of $L_{\mathrm{c}}$, the experimental coupling loss follows the same trend as the calculated values, with an offset of approximately $1 \mathrm{~dB}$ that we attribute to systematic deviations from the ideal geometry that was modeled in the numerical simulations.

The observed improvement of the coupling efficiency cannot be attributed exclusively to the gradual decrease of mode size and increase of mode index provided by the additional MISI section. Indeed, if we consider the effective index of the fundamental plasmonic mode $\left(n_{\mathrm{eff}}=2.51\right)$, as well as the overlap integrals of the MISI mode with the fundamental mode of the silicon waveguide $\left(\Gamma_{\text {SOI-MISI }}=0.63\right)$ and the fundamental MISM mode $\left(\Gamma_{\text {MISI-MISM }}=0.75\right)$, the estimated coupling loss from the waveguide transitions is the sum off $10 \log \left(T_{\text {SOI-MISI }}^{\text {Fresnel }} \Gamma_{\text {SOI-MISI }}\right)$ and $10 \log \left(T_{\text {MISI-MISM }}^{\text {Fresnel }}\right.$ $\left.\Gamma_{\text {MISI-MISM }} e^{-\alpha_{\text {MISI }} L_{\mathrm{c}}}\right)$, or $3.8 \mathrm{~dB}$, which is, in fact, slightly higher than the loss calculated by FDTD for direct coupling. This level of coupling loss is indicated in Fig. 4 as a reference. 


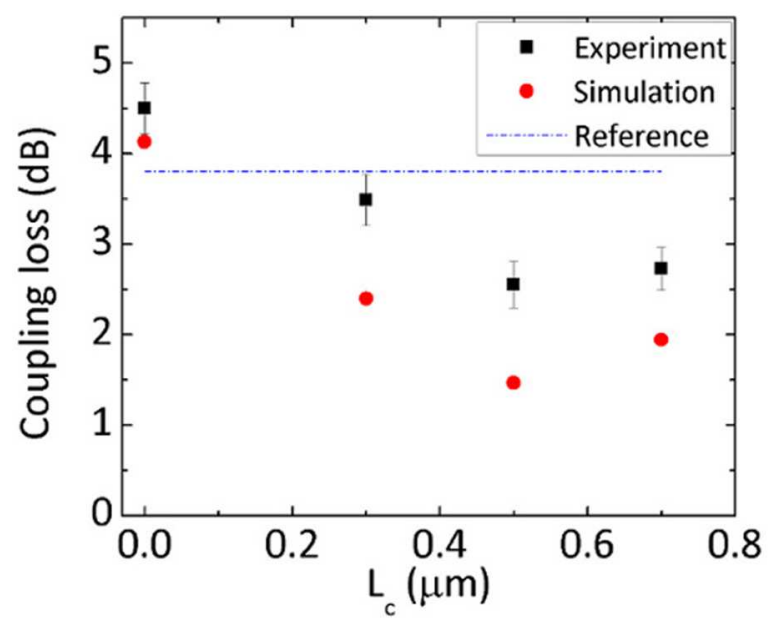

FIG. 4. Experimental (black squares) and simulated (red circles) coupling loss for different coupling lengths $\left(\mathrm{L}_{c}=0 \mu \mathrm{m}, \mathrm{L}_{c}=0.3 \mu \mathrm{m}, \mathrm{L}_{c}=0.5 \mu \mathrm{m}\right.$, $\left.\mathrm{L}_{c}=0.7 \mu \mathrm{m}\right)$. The reference loss is the value estimated for the waveguide transitions alone, neglecting interference effects.

The decrease in coupling losses for $L_{\mathrm{c}}=0.5 \mu \mathrm{m}$ is related to the oscillatory behavior observed in Fig. 2(c). Fabry Perot oscillations of plasmonic fundamental modes in the MISI and the MISM are expected to be very weak because (i) the reflection coefficients are small at both the SOI-MISI $\left(R_{\mathrm{SOI}-\mathrm{MISI}}^{\text {Fresnel }}=0.02\right)$ and MISI-MISM $\left(R_{\text {MISI-MISM }}^{\text {Fresnel }}=0.02\right)$ interfaces, and (ii) the modes are very lossy, especially for the MISM structure. Moreover, their periodicity would be, respectively, $\lambda_{0} / 2 n_{\mathrm{eff}}^{\mathrm{MISI}}=310 \mathrm{~nm}$ and $\lambda_{0} / 2 n_{\mathrm{eff}}^{\mathrm{MISM}}=225 \mathrm{~nm}$, where $\lambda_{0}=1550 \mathrm{~nm}$, which is very different from the periodicity of $1.5 \mu \mathrm{m}(0.8 \mu \mathrm{m})$ observed as $L_{\mathrm{c}}\left(L_{\mathrm{MISM}}\right)$ is varied (see Fig. 2). The observed oscillations of the attenuation are instead related to a multimode interference effect between either the MISI or the MISM fundamental plasmonic mode, and modes support at the lateral edges of the copper slabs (not shown), which have an effective index of approximately 1.55 for the MISI structure and 1.56 for the MISM structure, leading to an expected beating period of $L_{\text {MISI }}^{\text {beat }}=\lambda_{0} /\left(n_{\mathrm{eff}}^{\mathrm{MISI}}-1.55\right)$ $=1.6 \mu \mathrm{m}$ and $L_{\text {MISM }}^{\text {beat }}=\lambda_{0} /\left(n_{\text {eff }}^{\mathrm{MISM}}-1.56\right)=0.83 \mu \mathrm{m}$, in agreement with the observed periods of oscillation. These edge modes are mainly supported by the gate copper slab, the ones supported by the back contact copper slab being too lossy because of the presence of TiN. Reducing the contact thicknesses would change the modal effective index of the edge mode, so the beating period would change and cause a shift in the ideal coupler length.

In conclusion, we have demonstrated an efficient and straightforward approach for coupling light from a silicon waveguide to a vertical MISM plasmonic waveguide by inserting an intermediate MISI coupler of optimal length. The device is fully compatible with CMOS microelectronics circuitry and fabrication processes. This coupling scheme provides experimentally measured coupling loss as low as $2.5 \mathrm{~dB}$ per coupler for a coupler length of only $0.5 \mu \mathrm{m}$. The observed attenuation is a factor of three smaller than the value measured for directly coupled photonic and plasmonic waveguides, and is attributed to multimode interference effects between the waveguide mode and the plasmonic edge mode within the short coupling structure.

This work has been supported by the French National Agency (ANR) through LETI Carnot Funding. We acknowledge the LETI CMOS platform team for assistance in sample fabrication and K. Gilbert for technical contribution to the optical experiments.

${ }^{1}$ M. Lipson, J. Lightwave Technol. 23, 4222 (2005).

${ }^{2}$ R. Soref and B. Bennett, IEEE J. Quantum Electron. 23, 123 (1987).

${ }^{3}$ M. Ziebell, D. Marris-Morini, G. Rasigade, J.-M. Fédéli, P. Crozat, E. Cassan, D. Bouville, and L. Vivien, Opt. Express 20, 10591 (2012).

${ }^{4}$ R. Zia, J. A. Schuller, A. Chandran, and M. L. Brongersma, Mater. Today 9, 20 (2006).

${ }^{5}$ J. A. Dionne, K. Diest, L. A. Sweatlock, and H. A. Atwater, Nano Lett. 9, 897 (2009).

${ }^{6}$ A. Emboras, A. Najar, S. Nambiar, P. Grosse, E. Augendre, C. Leroux, B. de Salvo, and R. Espiau de Lamaestre, Opt. Express 20, 13612 (2012).

${ }^{7}$ W. L. Barnes, A. Dereux, and T. W. Ebbesen, Nature 424, 824 (2003).

${ }^{8}$ G. Veronis and S. Fan, Opt. Express 15, 1211 (2007).

${ }^{9}$ R. M. Briggs, J. Grandidier, S. P. Burgos, E. Feigenbaum, and H. A. Atwater, Nano Lett. 10, 4851 (2010).

${ }^{10}$ L. Chen, J. Shakya, and M. Lipson, Opt. Lett. 31, 2133 (2006).

${ }^{11}$ C. Delacour, S. Blaize, P. Grosse, J. M. Fedeli, A. Bruyant, R. SalasMontiel, G. Lerondel, and A. Chelnokov, Nano Lett. 10, 2922 (2010).

${ }^{12}$ E. D. Palik, Handbook of Optical Constants 1, 294 (1985).

${ }^{13}$ A. Snyder and J. Love, Optical Waveguide Theory, Science Paperbacks (Chapman and Hall, London, 1983).

${ }^{14}$ C. Kopp, E. Augendre, R. Orobtchouk, O. Lemonnier, and J.-M. Fedeli, J. Lightwave Technol. 29, 1847 (2011).

${ }^{15}$ H. S. Lee, C. Awada, S. Boutami, F. Charra, L. Douillard, and R. Espiau de Lamaestre, Opt. Express 20, 8974 (2012). 